\title{
Test of a device solving the interface issues between tenax tubes and a gas sensor array in an exhaled breath analysis context
}

\author{
M. Santonico', G. Pennazza', R. Capuano', P. Brinkman ${ }^{3}$, P.J. Sterk3, A. D’Amico1, \\ $U$-BIOPRED study \\ ${ }^{1}$ Department of Electronic Engineering, University of Rome Tor Vergata; Via del Politecnico 1, 00133 \\ Rome, Italy, \\ santonico@ing.uniroma2.it \\ ${ }^{2}$ Center for Integrated Research - CIR, Unit of Electronics for Sensor Systems, "Università Campus \\ Bio-Medico di Roma"; Via Alvaro del Portillo 21,00128 Roma, Italy, \\ ${ }^{3}$ Department of Respiratory Medicine, Academic Medical Centre, University of Amsterdam, \\ Amsterdam, The Netherlands
}

\begin{abstract}
:
Tenax tubes are also used for exhaled breath sampling. Hence, their preconcentration ability is strategic to detect disease biomarkers down to ppt. Moreover Tenax tubes are also easy to be delivered and stored, thus increasing reproducibility and standardization of the sample collection method. Many studies on breath analysis are currently being performed with non-selective gas sensor arrays (dubbed electronic nose). Tenax tubes are commonly used with traditional instruments of analytical chemistry. This study proposes a desorbing unit for tenax tubes to be used with a gas sensors array, in order to gain reproducibility and lower limits of detection for this novel technique. Here this device is tested and validated on a limited number of breath samples from asthma patients.
\end{abstract}

Key words: Tenax Tubes, Gas Sensor Array, Exhaled Breath, Asthma.

\section{Introduction}

Exhaled breath sampling procedure still needs to be improved in terms of reproducibility, storage, delivery conditions and high resolutions requirements.

Tenax tubes represent a good option for these requirements [1], but they are more frequently and commonly used with traditional analytical chemistry instruments than with electronic nose. In fact, tenax tubes are suitable for Gaschromatographs and other instruments typical of the analytical chemistry, which are designed for the thermally management of these cartridges.

The aim of the present study is to develop and validate devices able to desorb into an electronic nose chamber the exhaled breath sampled by tenax tubes from asthmatic patients.

\section{Materials and Methods}

The electronic nose used in this crosssectional study was the most recent version fabricated at the university of Rome Tor Vergata, frequently and currently used in many medical applications [2] and for asthma diagnosis to [3].

Four asthma patients (meeting GINA criteria) and two healthy controls were selected by the Academic Medical Center of Amsterdam as pilot study for an European IMI project UBIOPRED (www.ubiopred.eu). Each patient was sampled twice, and their exhaled breath was collected into a tedlar bag (using the Standard Operating procedure of the UBiopred project). The content of each Tedlar bag was then transferred into a Tenax tube.

Two Principal Component Analysis (PCA) models were built on the data collected.

The first was addressed to show the differences between the responses registered by the electronic nose for an empty tenax tube, a not-loaded tenax tube, a not-loaded and notheated tenax tube, two tenax tubes loaded with the exhaled breath of two control individuals.

The second was addressed to discriminate between different asthma conditions. 
The heating temperature profile was from $50^{\circ} \mathrm{C}$ to $200^{\circ} \mathrm{C}$ with steps of $50^{\circ} \mathrm{C}$. Thus, for each of the sample measured, the whole apparatus (gas sensor array and tenax tubes desorber) give a pattern of 32 responses ( 8 sensors for each of the four temperatures).

\section{Results}

Figure 1 shows the effectiveness of the desorbing methods being the four samples well distinguishable one from each other. It is worth noting that heating of the not loaded tube does not show remarkable modifications of the original sample; this suggests that the thermally desorption can help to separate the different compounds family forming the exhaled breath mixture. Moreover, along Principal Component (PC) 1 the richness in compounds abundance seems to be indicated: in fact, the samples there represented by the clusters are referred to (starting from right to left) a reference empty tube, the same tube when heated and the tenax tube loaded with exhaled breath. PC3 seems to be correlated with the different composition of the two individual breath samples.

Figure 2 shows the results obtained calculating a PCA model on four different asthmatic subjects, with promising classification ability. In fact, along the PC1, it is possible to discriminate between allergic mild asthma and adult asthma. Moreover, PC1 seems also to indicate the direction (from left to right) of asthma severity, because the four patients represented by the four clusters are dislocated along the PC1 from the left (allergic asthma) to the right (severe asthma) passing through the adult asthma. It is worth remarking that temperature seems to be correlated with PC4, but the number of measurements is to low to define a clear role of desorption temperature in this diagnostic problem.

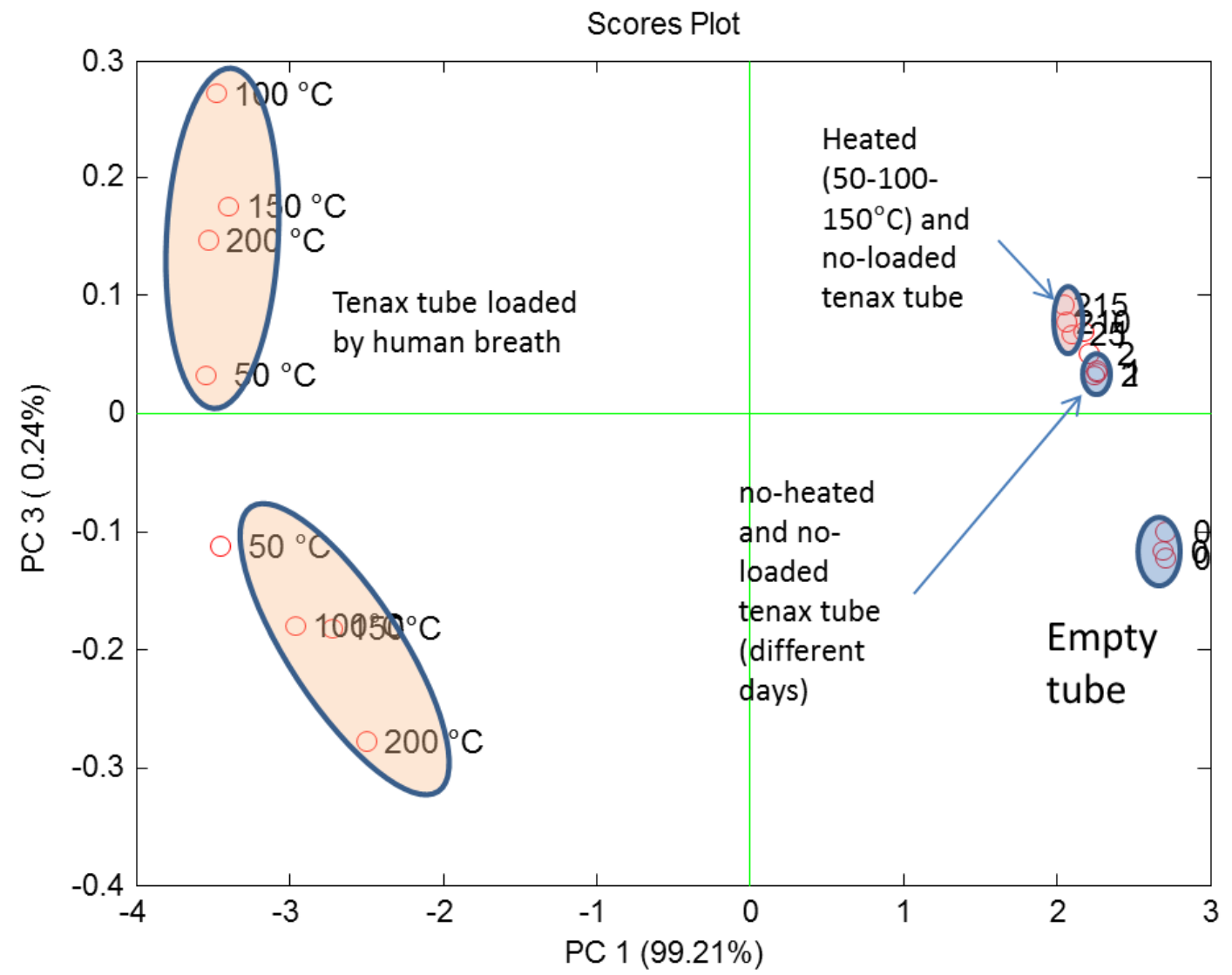

Fig. 1. : Scores plot of PC1 and PC3 of the PCA model built on the data registered by the electronic nose for an empty tenax tube, a not-loaded tenax tube, a not-loaded and not-heated tenax tube, two tenax tubes loaded with the exhaled breath of two control individuals. 


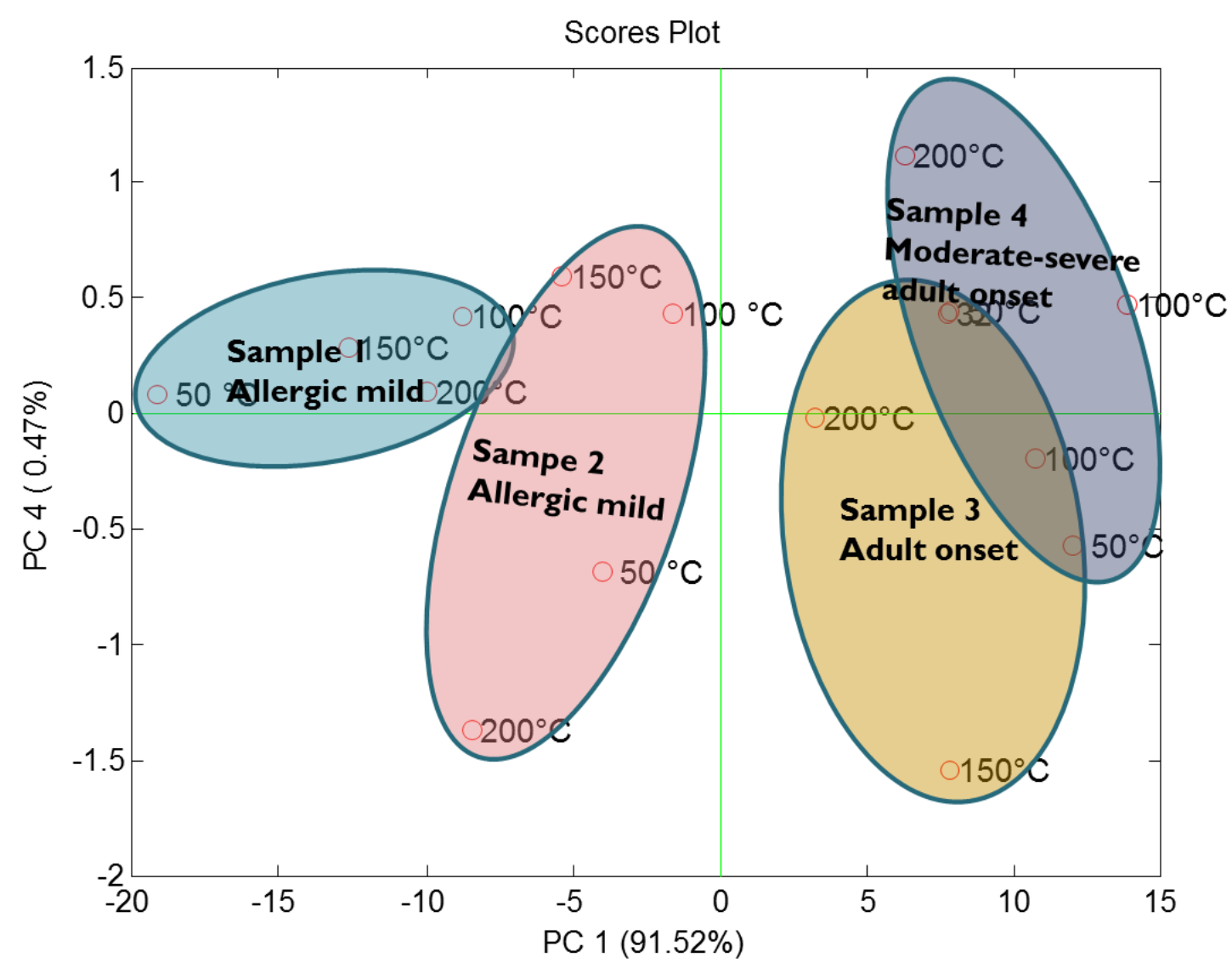

Fig. 2. : Scores plot of PC1 and PC4 of the PCA model built on the data registered by the electronic nose for four different asthmatic patients.

\section{Conclusions}

In the field of gas sensor arrays applied to diagnostic problems [2, 3] the innovative technique here proposed could represent an alternative to the spread used of the sampling bags for breath analysis. The effectiveness of this method accounts for the possibility of a practical utilization in a clinical experiment. Moreover, the tenax tubes thermal desorption here applied to a gas sensor array could open the way to a hybrid application of not selective chemical sensor with an analytical chemistry approach.

\section{References}

[1] Wzorek, B., Mochalski, P., Śliwka, I., \& Amann, A. (2010). Application of GC-MS with a SPME and thermal desorption technique for determination of dimethylamine and trimethylamine in gaseous samples for medical diagnostic purposes. Journal of Breath Research, 4(2).

[2] D’Amico A., Pennazza G., Santonico M., Martinelli E., Roscioni C., Galluccio G., Paolesse R., Di Natale C. (2010). An Investigation on electronic nose diagnosis of lung cancer. Lung Cancer, 68:170-176.

[3] Montuschi P., Santonico M., Pennazza G., Mondino C., Mantini G., Martinelli E., Capuano R., Ciabattoni G., Paolesse R., Di Natale C., Barnes P., D'Amico A. (2010). Diagnostic performance of an exhaled nitric oxide and lung function testing in asthma. Chest, 2010; 137(4):790-796. 